\title{
Evidence and advocacy: Are all things considered?
}

\section{Jeffrey A. Johnson, Alun L. Edwards}

$\mathrm{T}$ he recent exchange on the discord between influential recommendations is highly relevant to our health care systems. ${ }^{1-3}$ The Canadian Diabetes Association (CDA)'s national clinical practice guidelines (CPGs), past $^{4}$ and present, ${ }^{5}$ are among the most rigorous and evidencebased guidelines in Canada; ${ }^{6}$ yet, about $50 \%$ of the recommendations are based solely on consensus, with only the lowest level of evidence available (grade D) in the grading scheme. ${ }^{5}$ Although reaching consensus can be difficult, a consensus reached by one group, using one process, may differ from one reached by another group using a different process. ${ }^{7}$ This underscores the role of individuals and influences in the achievement of consensus, particularly in the absence of stronger evidence.

What is important in this ongoing exchange is not just about differences in consensus, but differences in mandate. It is not surprising that recommendations from groups such as the Canadian Expert Drug Advisory Committee (CEDAC) differ from those of CDA's CPG Expert Committee; their mandates are clearly different. The CDA should, however, recognize that its mandate "to advocate for and increase treatment options" is separate from its community-service mandate in creating evidence-based CPGs. Whereas CEDAC and the various provincial formulary-advisory committees must consider evidence of effectiveness as well as cost-effectiveness, ${ }^{3}$ CDA's CPG Expert Committee has explicitly excluded cost and costeffectiveness considerations from its deliberations. ${ }^{8}$ What is surprising is that developers of CPGs explicitly exclude considerations of cost and cost-effectiveness and then advocate that bodies such as CEDAC and the various provincial formulary-advisory committees should accept the CPG recommendations. This strategy is commonly used as well by pharmaceutical manufacturers in their argument for inclusion of their product on a formulary listing (e.g., "our product is listed in the CPGs, so therefore it should be available on formulary"). Ironically, by explicitly excluding cost and costeffectiveness considerations from the guideline development process, developers have provided formulary advisors an effective defence against this strategy (i.e., "Yes, but, those CPGs didn't consider cost or cost-effectiveness").

One strategy for attenuating this discord is the inclusion of cost and cost-effectiveness considerations in the development of CPGs. ${ }^{9,10}$ This need not take the form of 2 different competing recommendations, one based on clinical evidence, the $\vec{m}$ other based solely on cost; ${ }^{8}$ indeed, the consideration of both cost and effectiveness together form the basis of costeffectiveness analyses. If a new technology offers a clinical advantage (i.e., is safer or more effective) - which should be the first consideration - the question becomes, is that incremental benefit worth the incremental cost? Surely, if grade D consensus can be the evidentiary basis for half of the recom- mendations in the current version of the CDA's CPGs, there is room for the consideration of cost and cost-effectiveness in that process.

Regardless, collaboration and communication between CPG creators and policy-makers should be enhanced: The expertise of disease specialists might be coupled with economic and resource considerations in the creation of specific limitations for funded therapies (e.g., as suggested by the CDA in its recommendations for insulin glargine). Although the creation of "limited use" or "special authorization" criteria may, to practising physicians, seem unduly bureaucratic, to many the subtleties of grade D consensus guidelines ("therapy might be considered") are frequently not perceived and often abused by advocates and marketing forces.

\section{The concept of evidence- based medicine could be} extended into evidence-
based policy.

Given the recent growth of health care expenditure and its status as the largest portion of overall provincial spending, to ignore cost considerations seems a naive approach to advocacy. Consider, for example, an organization advocating for resource allocation for technologies and services that are recommended on the basis of grade D consensus. In the absence of stronger evidence, such products and services may be less effective, ineffective or, worse, potentially harmful. Advocates for such technologies and services would, in fact, be doing a disservice to their constituency, by potentially siphoning resources away from interventions already proven effective, from which patients would gain the greatest benefit; this is the concept of opportunity cost.

As a community, clinicians have embraced evidence-based medicine, and we hope that this concept can be extended into evidence-based policy. Policy advisors and participants in committees that develop CPGs need to work together and share responsibility for considering affordable health care solutions. They have the expertise to recognize which products and services should be accorded the highest priorities. ${ }^{2}$ They should consider a strategy of evidence-based advocacy, judiciously encouraging access in our health care systems to the interventions and services with evidence of the greatest benefit, rather than following an approach that simply advocates for all products and services currently available. 
This article has been peer reviewed.

From the Department of Public Health Sciences, University of Alberta and the Institute of Health Economics (Johnson), Edmonton, and the Department of Medicine (Edwards), University of Calgary, Calgary, Alta.

Competing interests: Jeffrey Johnson has received research grant funding from the Canadian Diabetes Association (CDA), Eli Lilly, Sanofi-Aventis, Glaxo SmithKline and Pfizer. He has supervised students who have received CDA Doctoral Research Awards, has served on the CDA Personnel Awards Committee (2003-2006) and CDA Applied Research Grants Committee (2002-2006), and is currently a member of the Expert Committee on Drug Evaluation and Therapeutics for Alberta Health and Wellness. Alun Edwards has acted as an investigator for several industry-funded trials and (before 2003) received speaker's fees for educational presentations. Both authors are participating in the revisions to the CDA's clinical practice guidelines to be published in 2008.

Contributors: Both authors contributed to the concept and writing of this commentary and have reviewed the final draft.

Acknowledgements: Jeffrey Johnson holds a Government of Canada Research Chair in Diabetes Health Outcomes and is a Health Scholar with the Alberta Heritage Foundation for Medical Research. The views expressed in this article are those of the authors and do not necessarily represent the views of other members of Alberta's Expert Committee on Drug Evaluation and Therapeutics.

\section{REFERENCES}

I. Clinical practice guidelines and conflict of interest [editorial; published erratum in CMAJ 2005;174(I):167]. CMAJ 2005;173(II):1297, I299.

2. Howlett MC, Lillie D. The Canadian Diabetes Association guidelines: putting the evidence first. $C M A J$ 2006;174(3):333-4.

3. Laupacis A. On bias and transparency in the development of influential recommendations. $C M A J$ 2006;174(3):335-6.

4. Canadian Diabetes Association. 1998 clinical practice guidelines for the management of diabetes in Canada. CMAJ I998;159(Suppl 8):SI-29.

5. Canadian Diabetes Association Clinical Practice Guidelines Expert Committee. 2003 clinical practice guidelines for the prevention and treatment of diabetes. Can J Diabetes 2003;27:Suppl 2.

6. Graham I, Beardall S, Carter AO, et al. What is the quality of drug therapy clinical practice guidelines in Canada? CMAJ 2001;165(2):157-63.

7. Leape LL, Park RE, Kahan JP, et al. Group judgments of appropriateness: the effect of panel composition. Qual Assur Health Care 1992;4:151-9.

8. Harris SB, McFarlane P, Lank CN. Consensus, cost-effectiveness and clinical practice guidelines [letter response]. Can J Diabetes 2005;29:376-8.

9. Ramsey SD. Economic analyses and clinical practice guidelines: Why not a match made in heaven? J Gen Intern Med 2002;17:235-7.

Io. Wallace JF, Weingarten SR, Chiun-Fang C, et al. The limited incorporation of economic analyses in clinical practice guidelines. J Gen Intern Med 2002;17:210-20.

Correspondence to: Dr. Jeffrey Johnson, Institute of Health

Economics, 10405 Jasper Ave., Rm. I200, Edmonton AB T5J 3N4; jeff.johnson@ualberta.ca

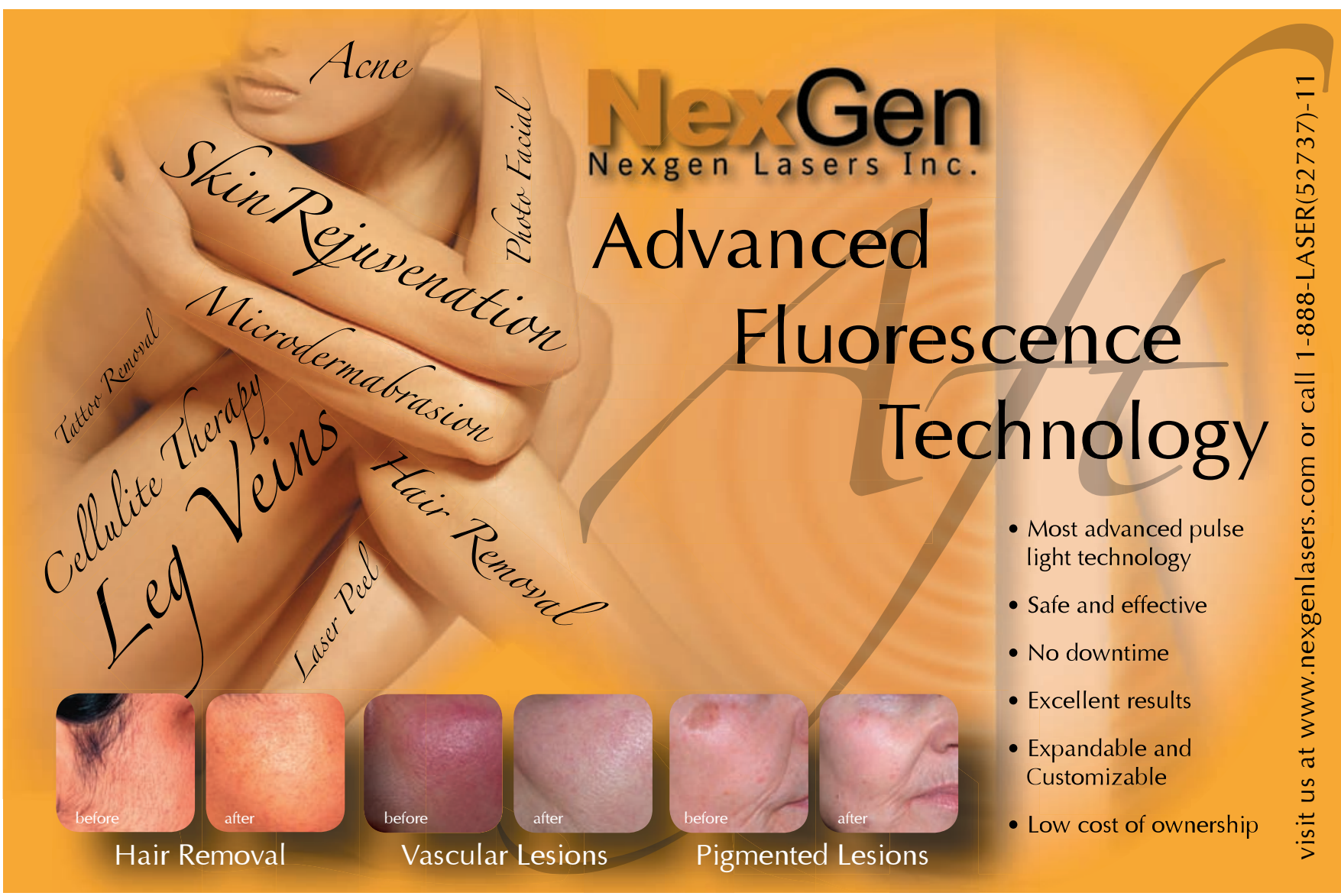

